A. Pramesh Rao, G. Swarup and Gopal-Krishna, eds.

\title{
A VLA Search for the Geminga Pulsar at 74 and $326 \mathrm{MHz}$
}

\author{
T. Joseph W. Lazio and Namir E. Kassim \\ Naval Research Laboratory, Code 7213, Washington, DC 20375-5351 \\ $U S A$
}

\begin{abstract}
We have used the VLA to image the location of the gammaray pulsar Geminga at 74 and $326 \mathrm{MHz}$. Upper limits to the pulseaveraged flux density, taking diffractive scintillation (DISS) into account, are $S<56 \mathrm{mJy}(2 \sigma ;$ DISS quenched) at $74 \mathrm{MHz}$ and $S<5 \mathrm{mJy}(95 \%$ confidence) at $326 \mathrm{MHz}$. Intrinsic variability appears to be required in order to reconcile our upper limits with previous low-frequency detections.
\end{abstract}

\section{Introduction}

Though initial speculation included the possibility that Geminga, the compact gamma-ray source in the Galactic anticenter, was a pulsar, its identification as such was not secure until the detection of X-ray pulsations (Halpern \& Holt 1992; Bertsch et al. 1992). Since most pulsars are detectable at radio wavelengths, intense effort has been devoted to finding a radio counterpart to Geminga.

Until recently, only upper limits on Geminga's radio emission could be established. A number of groups have now reported pulsed emission near $100 \mathrm{MHz}$ (Kuz'min \& Losovskii 1997; Malofeev \& Malov 1997; Shitov \& Pugachev 1997; Vats et al. 1999), though subsequent attempts at 35 and $326 \mathrm{MHz}$ failed (Ramachandran et al. 1998; McLaughlin et al. 1999). In an effort to constrain further the spectrum of Geminga, we conducted 74 and $326 \mathrm{MHz}$ observations (Kassim \& Lazio 2000)

\section{Observations}

The observations were conducted with the A-configuration VLA. Ionospheric variations would have vitiated a periodicity search, so only imaging observations were conducted. Calibration was performed within AIPS, using procedures similar to those for high-frequency data. One important aspect of imaging with the VLA at low frequencies is the sky cannot be approximated as flat. This "3-D" problem is severe at low frequencies because the primary beam is large. We used a polyhedron algorithm (Cornwell \& Perley 1992) in which the sky is approximated by many 2-D "facets." Figure 1 shows our $74 \mathrm{MHz}$ image of Geminga's location. 

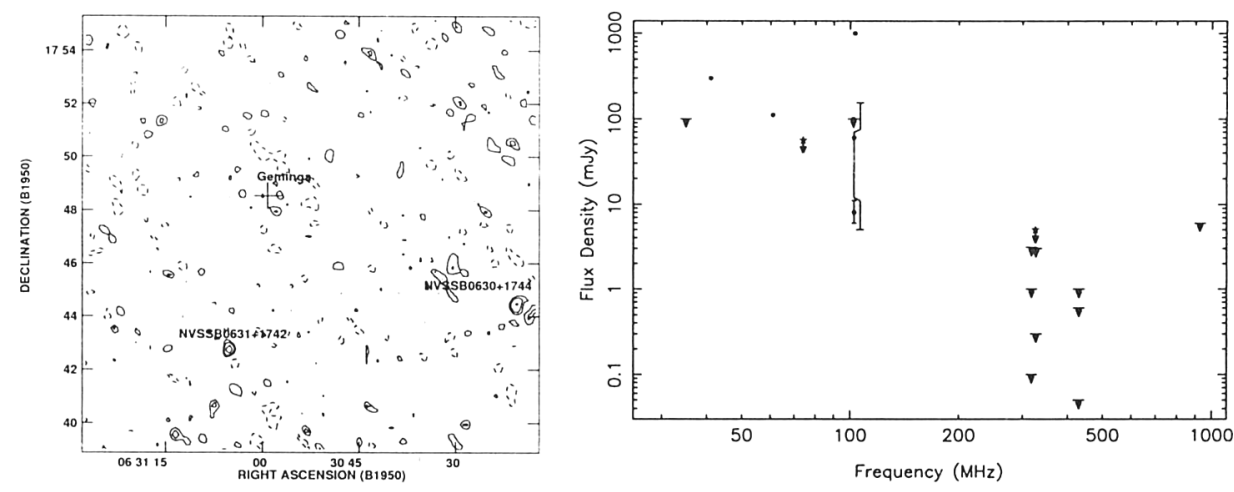

Figure 1. (Left) Geminga at $74 \mathrm{MHz}$. The angular resolution is $25^{\prime \prime}$, and the rms noise level is $28 \mathrm{mJy} \mathrm{beam}^{-1}$. The cross marks the location of the optical counterpart (Caraveo et al. 1998), and the sources NVSS B0630+1744 and B0631+1742 are marked for reference. The $326 \mathrm{MHz}$ image is similar. (Right) The spectrum of Geminga.

\section{Scintillation and Upper Limits on Geminga's Flux Density}

Figure 1 shows the spectrum for Geminga below $1000 \mathrm{MHz}$, combining our upper limits with measurements in the literature. In establishing our upper limits, we accounted for the effects of diffractive interstellar scintillation by following the procedure developed by McLaughlin et al. (1999). For our observational parameters, DISS is quenched at $74 \mathrm{MHz}$. We therefore adopt $S<56 \mathrm{mJy}$, twice the rms noise. In contrast, DISS has a substantial impact at $326 \mathrm{MHz}$. The upper limit is $S<5.0 \mathrm{mJy}$ (95\% confidence).

\section{References}

Bertsch, D. L., et al. 1992, Nature, 357, 306

Caraveo, P. A., et al. 1998, A\&A, 329, L1

Cornwell, T. J. \& Perley, R. A. 1992, A\&A, 261, 353

Halpern, J. P. \& Holt, S. S. 1992, Nature, 357, 222

Kassim, N. E. \& Lazio, T. J. W. 2000, ApJ, 527, L101

Kuz'min, A. D. \& Losovskii, B. Ya. 1997, Astron. Lett., 23, 283

Malofeev, V. M. \& Malov, O. I. 1997, Nature, 389, 697

McLaughlin, M. A., et al. 1999, ApJ, 512, 929

Ramachandran, R., Deshpande, A. A., \& Indrani, C. 1998, A\&A, 339, 787

Shitov, Yu. P. \& Pugachev, V. D. 1997, New Astron., 3, 101

Vats, H. O., et al. 1999, MNRAS, 302, L65

Previously published in "Pulsar Astronomy - 2000 and Beyond", ASP Conf. Series,

202, 239, 2000, Eds: Kramer, Wex \& Wielebinski 